\title{
Research on the Formation and Development Mechanism of Innovative Cluster based on Complex Network
}

\author{
ZHA Wei ${ }^{1, \text { a }}$ \\ ${ }^{1}$ Hubei University of science and technology,Xianning, Hubei,437100 China \\ azhawei@163.com
}

Keywords: innovative cluster network; BA model; preferential connectivity; knowledge spillover

\begin{abstract}
Formation and evolution of innovative network is the core problem of research on innovative cluster theory, complete comprehension on formation and evolution rule of innovative network has important significance for cultivating and developing innovative cluster. Innovative cluster network is mainly composed of network node and network connection, innovative network structure made by direct or indirect network connection between nodes becomes complicated. Meanwhile, formation and evolution of innovative cluster network is also one complicated process, it is difficult to make clear its formation and evolution process. Scale-free model has growth and preferential connectivity, which can well simulate self-organization evolution process of innovative cluster network. On the basis of completely analyzing connection relation of innovative cluster network, this paper analyzes and demonstrates formation and evolution process of innovative cluster network, puts forward corresponding countermeasure and suggestion according to research conclusion.
\end{abstract}

\section{Introduction}

With the gradual increase in global economy, every country is actively promoting development road relies on technology advancement and research innovation. Cultivating innovative cluster is the necessary choice to increase cluster innovation ability, promote economic development and enhance national competition. Making research on formation of innovative cluster can make preparation for cultivating innovative cluster in the future. This paper starts from formation route of innovative cluster and element effect in the formation process, establishes system dynamics flow of innovative cluster formation mechanism and makes simulation on model. In the theoretical research of innovative cluster, innovative cluster network is always the focus researched by many scholars. Cluster definition determines innovative cluster has typical network characteristics. Innovative network is the important base to understand and make research on innovative cluster, it is also the core problem to cultivate and develop innovative cluster, applying complicated network theory to analyze evolution process of innovative cluster can make research on changes in network structure and function of innovative cluster in the different stages, promote and optimize coordination and interaction among innovation object, accelerate healthy and orderly development of innovative cluster.

\section{Complicated network characteristic of innovative cluster}

Innovative cluster network is the complicated system composed of many kinds of activity subjects, because of difference in scale, quantity and innovation ability in innovation subject as well as network relation among subjects, which causes innovative cluster have complicated network characteristics.

Firstly, subject of innovative cluster has diversity and heterogeneity. The innovation subject in innovative cluster includes enterprise, higher university, research institute, government, 
intermediary organ and financial organ etc, target, ability, resources, technological level of different subjects are also different, which causes diversity and heterogeneity in network node.

Secondly, related complication of innovative cluster among innovation subjects causes complicated network structure. Innovation subject has large quantity, which causes its network connection way become diversified and complicated. Networking among different subjects not only has formal relations established by agreement and contract etc, but also has informal relations based on trust. Complicated relation among innovation subject makes network structure of innovative cluster have complication and diversity.

Thirdly, network structure of innovative cluster has multi-level, because innovation subject is placed in the different positions, which causes connection degree and connection frequency different from other innovation subjects, this constructs multi-level network structure in innovative cluster network. Innovative network has organizations with different levels, network in the top embeds several low-level network and several innovative sub-cluster is divided according to industrial field and innovation direction, meanwhile each subject in the same innovative cluster can be divided into different network module according to different functions.

Fourthly, innovative cluster has dynamic and space evolution characteristics. Innovative cluster is one kind of network organization in order to cope with complicated and changeable external environment to realize its development target, so relations among nodes in cluster will dynamically change with changes in time and space to continually adapt to external economic and social environment.

\section{Formation process of innovative cluster network}

Speaking in theory, as one kind of organization form between industry and market, formation and evolution of innovative cluster should obey rule in certain time and space. According to present research outcome, one kind of mainstream opinion relies on innovative life cycle theory to make division on innovative cluster, this formation and evolution of innovative cluster can be roughly divided into 4 stages such as formation stage, development stage, mature stage and depression stage.

Formation of innovative subject is one relatively complicated process, there are more and more scholars start using scale-free network model to make analysis on innovative cluster network. This model has 2 characteristics: the first one is growth, which means overall attraction on organization beyond of core and element resources through agglomeration effect, so that this core node has more opportunity to allocate resources and it indicates bigger growth. The second is preferential connection, which means the same industry beyond industry or strong associated enterprises tends to regard as one node connected to assemble point which has more connection points and good growth. The formation process of innovative cluster network forms new network by adding point, side, and re-connection on the basis of initial network. We suppose initial state of innovative network is as follows: limited $\mathrm{m}_{0}$ nodes is randomly connected to form scheme, the set of node in the scheme $\mathrm{N} 0=\left\{1,2, \cdots, \mathrm{m}_{0}\right\}$ and $3 \square \mathrm{m}_{0} \mathrm{D} 100$, and $\mathrm{i}$ and $\mathrm{j}$ are 2 nodes in the set, which represents innovative subject in the innovative network, side $\mathrm{ij}$ indicates connection among subjects, $\mathrm{k}_{\mathrm{i}}$ indicates value of node $i$. When enterprises establishes connection with other enterprises in the cluster, under precondition of difference in moderate knowledge, it will choose partnership with big influence, which means choosing enterprises with more connections, it is the so-called preferential link mechanism, the mathematical formula can be indicted as follows:

$$
p\left(k_{i}\right)=\frac{k_{i}}{\sum_{j \in g_{t}} k_{j}}(1)
$$

Formula (1) indicates that in scheme $\mathrm{g}$ formed by $\mathrm{m}_{0}$ initial nodes, when it uses probability side, it will randomly choose one node as start point of this new side, choice of the other terminal is chosen by preferred connection mechanism. In the following, it will use preferred connection mechanism to describe formation process of innovative cluster network. 
(1)Adding side. Adding side means forming new connection with the existing nodes in network. Suppose it uses probability p to add m sides, it randomly chooses one node as start point as this new side; the other terminal repeats the following process for $m$ times according to formula (1).

(2)Re-connection. Re-connection means disconnecting existing connection and forming new connection. Re-connection usually takes place when one node finds existing connection has low profit and it has connection with higher profit. It uses probability q to reconnect $\mathrm{m}$ sides, firstly it chooses one node $i$ and one side $l_{i j}$ from this node, so it forms node $i$ and link $l_{i j}$ after disconnecting this side, probability of chosen probability will also conform to formula (1), the probability of choosing $\mathrm{j}$ will also conform to formula (1), and then repeat the following process for $\mathrm{m}$ times.

(3)Adding point. Adding point means network appearing new node and adding side starts from new side. It uses probability of $1-\mathrm{p}-\mathrm{q}$ to add one new node, new node and existing $\mathrm{m}$ nodes forms new connection, connection probability will also conform to formula(1).The above-mentioned process will make value of node have corresponding changes:

$$
\begin{aligned}
& \left(\frac{\partial k_{i}}{\partial t}\right)_{(1)}=p m \frac{1}{N}+p m \frac{k_{i}}{N}+p m \frac{k_{i}}{\sum_{j \in N} k_{j}} \\
& \left(\frac{\partial k_{i}}{\partial t}\right)_{(2)}=-q m \frac{1}{N}+1 m \frac{k_{i}}{N}+p m \frac{k_{i}}{\sum_{j \in N} k_{j}} \\
& \left(\frac{\partial k_{i}}{\partial t}\right)_{(3)}=(1-p-q) m \frac{k_{i}}{\sum_{j \in N} k_{j}}
\end{aligned}
$$

Combine with formula (1), (2) and (3), it can conclude the following:

$$
\left(\frac{\partial k_{i}}{\partial t}\right)=(p-q) m \frac{1}{N}+m \frac{k_{i}}{\sum_{j \in N} k_{j}}
$$

Power law distribution and index distribution can appear in network, network structure relies on network participation. Degree distribution of model network conforms to power law distribution of $\gamma=3$. The above-mentioned BA model finds out one simple and reasonable formation mechanism for innovative cluster network, which relatively simulates evolution process of self-organization network: innovative cluster is one non-linear connection network system among one vast innovation subjects, core node will produce attraction on geographical space in the same industry or relevant enterprise beyond of code node, these enterprises preferentially approach to node with more connection resources and forms new connection relations. This, under effect of growth and preferred connection mechanism, core node gradually expands to high-dense cluster system with plenty of connection resources, which is innovative cluster (such as figure 1 and figure 2).

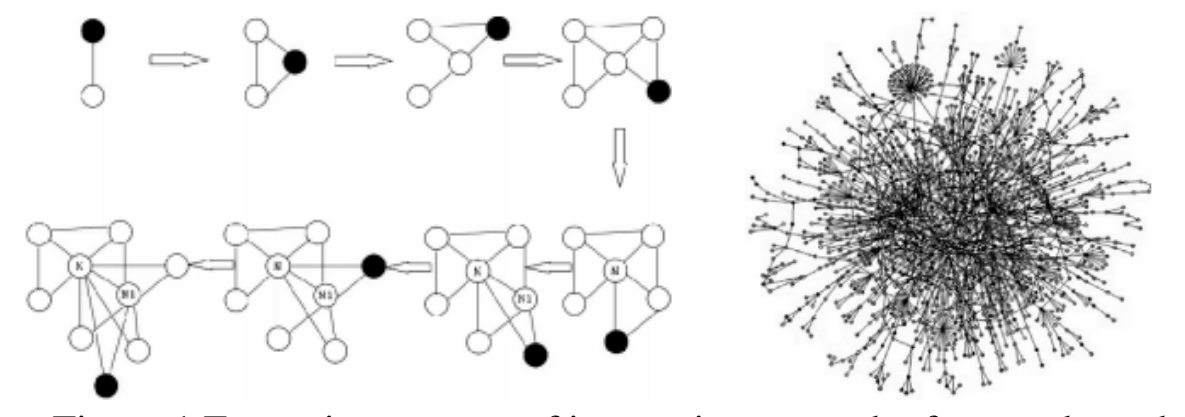

Figure 1 Formation process of innovative network of network model

Figure 2 Preferred connection mechanism derives from complicated innovative cluster network

\section{Simulation result and explanation}

In the detailed simulation process, this paper respectively considers network evolution process of innovative cluster under 2 different kinds of conditions. Firstly, under condition without 
interruption from government, enterprise, higher university or research institute connects to network by certain probability, establishes relation with present node, which makes network dynamically evolve. Secondly, government enters network by certain probability, this paper thinks government interruption will have vital effect on formation and evolution of innovative cluster; therefore, government participation will form strong connection in network and strengthen communication, cooperation of all subjects. Top point has difference in divergence degree distribution, divergence weight distribution and side weight distribution when $\mathrm{N}=100,200300,400,500$, meanwhile, under condition of different node quantity, it respectively investigates effect condition on network cluster coefficient and the average shortest by external force degree of government. Speaking in details, when $\mathrm{q}=1$, government dose not interrupt innovative cluster, when q takes $0.8,0.6,0.4$, interruption degree of government on cluster will gradually strengthen, including measures such as issuing policy, making plan, fund guidance etc to encourage enterprise, higher university and research institute to implement new cooperation, construct cooperation platform for industry, school and research. The first step: when $\mathrm{N}=100$, formation process of network, relation among nodes is relatively loose. The number of node in innovative network is 100 , enterprise node participates in network by larger proportion, higher university and research institute participates by low proportion, then it gets point-weight distribution, degree distribution, degree-weight relation and side-weight distribution, which is indicated by figure 3 and figure 4 .
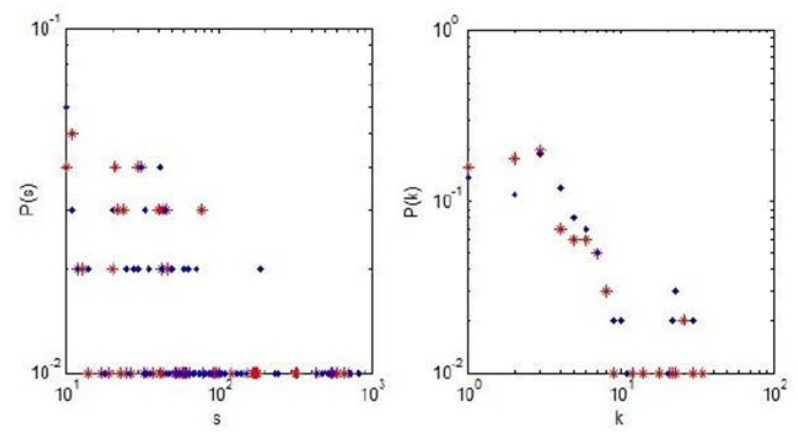

(a) Divergence distribution of top point (b) divergence distribution of top point

Figure 3 top point divergence weight and top point divergence degree of network when $3 \mathrm{~N}=100$

From figure 3 it can be seen that the top point connection degree of corresponding innovative cluster (surplus of exit and entry degree is total top point degree) mostly ranges from 0 to 5 , node which ranges connection degree from 0 to 5 accounts for larger probability, at this time, network connection degree in the whole network is not so high, there is only few nodes have higher connection degree, meanwhile, there are also a few nodes have low connection degree. This is basically corresponds to characteristics of innovative cluster in the formation stage, which means it has little node quantity in the whole innovative cluster network, connection degree is low, it is the formation stage of the whole network, every subject is in the networking process, it is the formation stage of innovative cluster.
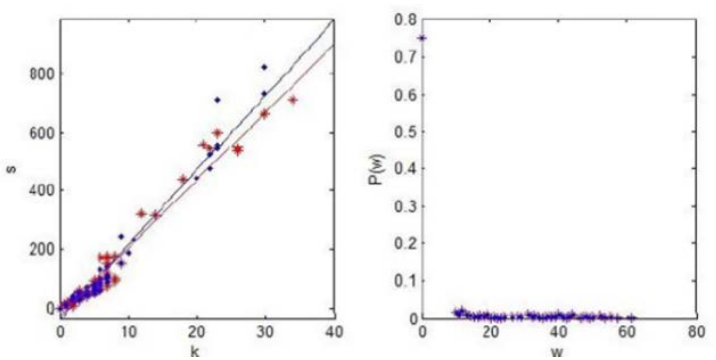

Figure 4 degree-weight relation and side-weight distribution scheme when $4 \mathrm{~N}=100$

Figure 4 describes relations between exit degree and exist degree weight, entry degree and entry degree weight, it can find that side weight of every side on average is 22.5(slope), which indicates entry side weight and exit side weight is stabilized at 22.5 or so, it mainly focuses on node with degree from 0 to 10, the probability of side-weight distribution is very large. In network, node has 
connection degree value of 5 will have the largest appearance probability, while node with connection degree of 40 or so is very few, this indicates self-innovative ability of cluster at this time mainly relies on single innovation ability of node itself, relation degree of every node in cluster is relatively low, the overall innovation ability in the whole innovative cluster has not formed.

\section{Conclusions}

This paper puts forward the following countermeasures and suggestions on cultivating and developing innovative cluster:

(1)Connection relation of innovative cluster network mainly includes industrial chain, innovative chain, fund chain and information chain, whether it can successfully cultivate innovative cluster or not, the key point lies in whether it can get through relations of 4 chains among enterprises. Government can get through 4 kinds of chains by adjusting industrial plan, perfecting market system, establishing public service platform such as cooperation by government, industry, school and research, innovative alliance, science finance etc.

(2) Formation of innovative cluster has preferential connection rule, code nodes with plenty of connection relations (innovative leading enterprises) have obvious attraction and agglomeration effect, meanwhile, knowledge spillover effect caused by code node and technology diffusion effect can effectively enhance innovation ability and industrial competition of the whole innovative cluster. Therefore, government should pay high attention to cultivation and support on leading enterprises in cluster; completely display acceleration and agglomeration effect.

(3) Whether enterprises participate in innovative cluster or not, the root lies in whether it can obtain excessive profit by innovative cluster, while this profit realization depends on whether knowledge information can quickly communicate and spill over in innovative cluster, and whether this enterprise have simulation and learning ability. Therefore, in the process of cultivating and developing innovative cluster by government, the key dose not lie in how to attract investment and enterprises, but lies in promoting and establishing platform and environment for communication, sharing and learning in the cluster, promote enterprises to enhance ability in learning, simulation and innovation.

(4) Innovative network dose not have radiation and enhancement effect on this cluster, but also has obvious radiation and acceleration effect on surrounding cluster. Practice demonstrates innovative cluster has already become to be the important engine for regional economy transformation and updating, government organ should pay high attention to cultivation and development of innovation cluster, make research and formulate special development plan and support policy.

\section{References}

[1] Xu Weixiang, Chen Bin. Research on Innovation Performance of Innovative Cluster [J]. Economic Perspective, 2013, 10:89-95.

[2] Yan Weiwei, Hu Changping. Research on Cross-system Information Integration Demand and Acceleration Faces with Innovative Cluster [J].Intelligence Data, 2015, 03:63-67.

[3] Bai Suxia, Chen Jingan. Research on Industrial Cluster Evolves to Innovative Cluster [J].Economic System Reform, 2015, 03:114-117.

[4] Zhen Jie. Cooperation Model, Effect Factor, Pattern and Choice among Enterprises in Innovation Cluster [J]. Contemporary Finance \& Economics, 2013, 05:72-81.

[5] Wu Youke. From Industrial Cluster to Innovative Cluster- Research on Industry Updating in Fujian [J].Science Management Research, 2013, 12:7-12+22. 